\title{
Swiss Quality Award - Innovations in Healthcare
}

En 2012, la Fédération des médecins suisses (FMH) attribuera à nouveau le Swiss Quality Award conjointement avec la Société suisse pour le management de la qualité dans la santé (SQMH) et I'Institut pour la recherche évaluative en médecine (IEFM) de I'Université de Berne. Le projet récompense des pionniers qui ont su influencer positivement et durablement le domaine de la santé au travers de leurs innovations.

Varja Nikolic

Correspondance: FMH / DDQ

Elfenstrasse 18

CH-3000 Berne 15

Tél. 0313591111

Fax 0313591112
Le Swiss Quality Award 2011 a été un succès et plus de 100 projets ont été envoyés. Les trois organismes responsables ont donc décidé de décerner ce prix une nouvelle fois l'année prochaine. Ce faisant, ils veulent non seulement saluer l'engagement des lauréats, mais également attirer l'attention sur la grande diver-

\section{Même les petits projets ont de fortes chances de gagner!}

sité des démarches qualité: de nombreux projets sont mis en œuvre dans le domaine de la santé pour le bien des patients. Comme à l'accoutumée, le prix sera décerné dans les quatre catégories suivantes: management, sécurité des patients, technologie et empowerment. Chaque catégorie est récompensée par un montant de 10000 francs.

Le Swiss Quality Award comprend la qualité au sens large. Cela se voit non seulement dans les quatre catégories primées, mais également dans les différents domaines d'application des projets soumis. Tous partagent cependant le même objectif: améliorer la qualité des soins.

\section{Envoyez vos projets dès maintenant!}

Vous avez mis en œuvre un projet novateur en matière de gestion de la qualité dans votre clinique, votre cabinet médical ou votre entreprise? Alors n'hésitez pas à vous inscrire au Swiss Quality Award 2012. Même les petits projets ont de fortes chances de gagner! Le délai d'inscription est fixé au 29 février 2012.

Les prix du Swiss Quality Award seront décernés lors du Symposium national pour le management de la qualité dans la santé qui aura lieu le 15 mai 2012 à Berne; une date qu'il convient de réserver aujourd'hui déjà!

Vous pouvez vous inscrire en ligne à l'adresse suivante: www.swissqualityaward.ch. Vous y trouverez toutes les informations utiles. La procédure d'inscription a été légèrement modifiée, c'est pourquoi il importe de consulter le site même si vous avez déjà participé aux éditions précédentes.

\section{Swiss Quality Award}

INNOVATIONS IN HEALTHCARE

\section{ENVOYEZ VOS PROJETS AVANT LE 29 FÉVRIER 2012}

Le Swiss Quality Award récompense les meilleures innovations en matière de qualité dans le système de santé. Participez et enregistrez votre projet sur le site www.swissqualityaward.ch. 